\section{The end-of-life experience of patients with rare cancers and their caregivers}

\author{
Elizabeth Trice Loggers, 1,2 \\ Holly G. Prigerson ${ }^{3,4}$ \\ ${ }^{1}$ Group Health Research Institute, Group \\ Health Cooperative, Seattle, WA; \\ ${ }^{2}$ Fred Hutchinson Cancer Research Center, \\ Seattle, WA; ${ }^{3}$ Center for Psychosocial \\ Epidemiology and Outcomes, Dana-Farber \\ Cancer Institute, Boston, MA; \\ ${ }^{4}$ Department of Psychiatry, Brigham and \\ Women's Hospital, Boston, MA, USA
}

\section{Abstract}

Little is known about the end-of-life (EOL) experience of patients with rare cancers (PRC) or their caregivers. From September 2002 to August 2008, 618 stage IV cancer patients [195 PRC and 423 patients with common cancers (PCC)] and their caregivers participated in an interview-based cohort study. Patients were interviewed about EOL preferences, planning, medical care, and followed until death. Interviews with caregivers at baseline assessed caregiver mental and physical health; and postmortem, assessed EOL patient care. PRC were four times more likely than PCC to be receiving both radiation and chemotherapy at study entry ( $10.3 \%$ vs $3.3 \%$, respectively, adjusted odds ratio $4.31, \mathrm{P}=0.003)$. PRC's caregivers were more likely to report declining health $(22.1 \%$ vs $15.7 \%$, $\mathrm{P}=0.05$ ) and marginally more likely to report using mental health services to cope than PCC's caregivers. PRC were as likely to acknowledge their illness was terminal, have EOL discussions, and participate in advance care planning as PCC. Future research should investigate terminal care for PRC and how providing care affects caregivers' physical and mental health.

\section{Introduction}

One in 10 , or 25 million to 30 million, Americans have a rare condition (National Organization of Rare Diseases), which the Rare Diseases Act of 2002 defined as a disease with fewer than 200,000 prevalent U.S. cases per year (Rare Diseases Act of 2002). ${ }^{1}$ While each rare condition is uncommon, their collective impact may be substantial. ${ }^{2,3}$ The same is true for rare cancers, which represented $23.4 \%$ of all cancer cases and $32.9 \%$ of cancer deaths. ${ }^{4}$

Rare cancers have been defined as having fewer than 150 cases per million cancer cases per year. ${ }^{5}$ Regardless of the definition used, the difficulty of amassing cases frustrates understanding the etiology, diagnosis, and treatment of these tumors. ${ }^{6,7}$ In response, efforts continue to create national registries for rare cancers, pool tissue and data, develop effective randomized clinical trials, and create incentives for treatment and/or drug development. ${ }^{1,-14}$ Despite these initiatives, institutional lack of interest, disproportionately low funding, publishing biases, and unique ethical issues may hamper study of rare cancers., ${ }^{75-17}$

People with rare cancers and their families may also face significant challenges, such as physicians' lack of familiarity with the disease, diagnostic delays or errors, logistical difficulties including coordination among multiple specialists and visits, conflicting treatment recommendations, and a dearth of clinical or scientific evidence to guide decision-making. ${ }^{6,15,18-20}$ This may prompt feelings of alienation, bewilderment, and apprehension, ${ }^{19}$ or stress (e.g. fear, anxiety, and heightened worry). ${ }^{21,22}$ Alternatively, it can result in hope and optimism in the face of uncertainty about treatment outcomes. Either psychosocial context could affect treatment decision making and future appraisals of that decision making (i.e. regret) $)^{23}$ - and be of particular significance for the end-of-life (EOL) experience.

Rare cancers may represent significant psychosocial impact and risk of death for patients. ${ }^{6,24}$ Therefore, we were interested in whether, and how, the EOL experience among adult patients with rare cancers differed from that of individuals with common cancers. To address this question, we used the dataset of Coping with Cancer (CWC), a prospective, NCIfunded multi-site study of terminally ill cancer patients. However, we found little prior literature to guide our investigation. Therefore, based on clinical experience, we hypothesized that oncologists may communicate differently about rare versus common cancers. Specifically, given a general lack of proven treatments for rare cancers collectively, we speculated that a patient's terminal condition might be discussed more immediately and frankly. We hypothesized that, compared to patients with common cancers, rare cancer patients with advanced disease would be more likely to: i) receive care in a tertiary or referral cancer center, reflecting a perception by patients and caregivers that tertiary-center-based oncologists have more experience treating rare cancers or would have greater access to clinical trials; ii) acknowledge their illness was terminal; iii) participate in advance care planning and EOL discussions; but iv) want a more intensive or aggressive approach to EOL care in the face of uncertainty. We also hypothesized that at study entry caregivers of rare cancer patients would be more likely to: i) be emotionally distressed; ii) want more information about the cancer and treat-
Correspondence: Elizabeth Trice Loggers, Group Health Research Institute, 1730 Minor Avenue, Suite 1600 , Seattle, WA 98101, USA.

Tel. +1.206.442.5222 - Fax: +1.206.287.2871.

E-mail: loggers.e@ghc.org

Key words: end-of-life care, caregiver burden, terminal illness.

Contributions: HP data collection; EL, HP data analysis, manuscript writing and review.

Conflict of interests: the authors declare no potential conflict of interests.

Funding: this research was supported in part by the following grants to Dr. Prigerson: MH63892 from the National Institute of Mental Health and CA 106370 from the National Cancer Institute; and the Center for Psychosocial Epidemiology and Outcomes, Dana-Farber Cancer Institute.

Received for publication: 2 January 2014.

Accepted for publication: 6 February 2014.

This work is licensed under a Creative Commons Attribution NonCommercial 3.0 License (CC BYNC 3.0).

(C) Copyright E.T. Loggers and H.G. Prigerson, 2014 Licensee PAGEPress, Italy

Rare Tumors 2014; 6:5281

doi:10.4081/rt.2014.5281

ment options; and iii) want the patient to take an aggressive approach to treatment.

\section{Materials and Methods}

\section{Study sample}

Participants were adult patients with advanced cancer (defined as metastatic disease and failure of first-line chemotherapy) and their informal caregivers recruited as part of the CWC study. The specifics of the CWC study, its inclusion and exclusion criteria, and participation rates have been described in detail elsewhere. ${ }^{25}$ Briefly, of the 944 patients who were initially approached and confirmed to be eligible, 274 (29.0\%) declined participation. Compared to those who declined to participate, participants were less likely to be distressed on a 5 -point Likert scale whose extremes ranged from 1 (minimaVnonexistent) to 5 (distraught) (mean score 2.53 vs 2.94; $\mathrm{P}<0.001)$. Non-participants and participants did not differ significantly in sex, age, race, or education. The human subjects committee at each institution reviewed and approved all study protocol and contact documents before research was conducted; and all participants provided written informed consent. 
Cancer type for each patient was determined by chart review. Patients were classified as having rare or common cancers based on the American Cancer Society's 2009 estimate of new cancer cases and the cutoff for common cancers used by the National Cancer Institute (i.e. those cancers with more than $35-40,000$ incident cases per year) ${ }^{26}$ Cancers of unknown primary origin were classified as rare. This analysis includes 618 terminally ill cancer patients, including 195 (31.6\%) with rare cancers. Table 1 reports the types of rare cancer in the sample. The most frequent common cancer cases were: 149 (35.2\%) lung, 77 (18.2\%) colorectal, 76 (18.0\%) breast, and 49 (11.6\%) pancreatic cancer.

\section{Measures \\ Patient demographic and health measures}

Patients and caregivers participated in separate baseline interviews conducted by trained interviewers. Collection of sociodemographic data, health measures (including Karnofsky and Charlson), quality of life (McGill Quality of Life Questionnaire), mental health assessments (Structured Clinical Interview and Diagnosis) and treatment information for patients have been described elsewhere. ${ }^{25}$ All scales are psychometrically sound and have established reliability and validity. ${ }^{27-31}$

For this study, the designation of a tertiary or referral cancer center was applied to: Yale Cancer Center (New Haven, CT); Dana-Farber Cancer Institute and Massachusetts General Hospital Cancer Center, Boston (MA); and Simmons Comprehensive Cancer Center, University of Texas, Southwestern Medical Center, Dallas (TX). Non-tertiary centers included Veterans Affairs Connecticut Healthcare System, West Haven, (CT); Parkland Hospital Palliative Care Service, Parkland Health and Hospital System; and New Hampshire OncologyHematology, Hookset (NH).

\section{Caregiver measures}

Caregiver health was measured using the Medical Outcomes Study SF-36 survey. ${ }^{32}$ Caregiver mental health coping was assessed with the following yes or no question: Have you accessed any type of mental health intervention to help yourself adjust to the patient's illness since she/he was diagnosed with cancer? Caregivers also completed scales to assess their coping styles and traits including the Control and Optimism scale.

To assess doctor-patient communication and information giving, the following questions were asked (yes or no response options): i) Do you feel that (patient's) doctors here listen to your concerns about (patient's) medical treatment? ii) How much information do the doctors provide you with about (patient's) medical treatment? Would you say: less information than needed, more information than needed, or just the right amount. iii) Are there any services that you would like to see offered here at the hospitaVclinic to help you and your family cope with (patient's) illness? If so, what are they? Answers for this question were recorded verbatim.

With respect to caregiver care preferences, caregivers were asked: If you had to decide, would you prefer that (patient) chose: i) a course of treatment that focused on extending life as much as possible, even if it meant more pain and discomfort, or ii) on a plan of care that focused on relieving pain and discomfort as much as possible, even if that meant not living as long? Caregivers choosing extend life as much as possible were designated as preferring intensive EOL care.

\section{Patient end-of-life measures}

EOL patient conversations were assessed with the following yes or no questions: Have you completed a Do Not Resuscitate (DNR) order?, and Have you and your doctor discussed any particular wishes you have about the care you would want to receive if you were dying?

Participation in advance care planning was determined if the patient indicated they had either a health care proxy or living will or both. A preference for intensive EOL care was defined as answering Extend life as much as possible rather than Relieve pain or discomfort as much as possible to the following question: If you could choose, would you prefer: i) a course of treatment that focused on extending life as much as possible, even if it meant more pain and discomfort, or ii) on a plan of care that focused on relieving pain and discomfort as much as possible, even if that meant not living as long?

Caregivers participated in a post-mortem interview to determine the patient's EOL care. The EOL care outcomes of interest included whether a patient received intensive EOL care defined as CPR and/or ventilation in the last week of life followed by death in an intensive care unit, in- or outpatient hospice care, and location of death.

\section{Statistical analysis}

Descriptive statistics were used to characterize study sample demographics. T-tests were used for continuous variables, CochranMantel-Haenszel statistics for categorical variables, and chi-square statistics or Fisher exact tests for binary variables. Logistic regression was used to determine the association between cancer type and care received, adjusting for confounders (entered into the model at a significance level of $\mathrm{P}<0.10$ and retained at a significance level of $\mathrm{P}<0.05)$. All patient sociodemographic characteristics, health, quality of life, and communication variables were considered as potential confounders. Results are presented as unadjusted (UOR) and adjusted odds ratios (aOR). Statistical inferences were based on two-sided tests with $\mathrm{P}<0.05$ as the cutoff for statistical significance. Data analysis was conducted using SAS System for Windows v. 9.1 (SAS Institute, Inc., Cary, NC, USA).

\section{Results}

\section{Patient characteristics}

Table 2 shows the socio-demographic and clinical characteristics of the sample. Patients with rare cancers were on average two years younger, had fewer comorbidities, and were more likely to be Hispanic. The association of ethnicity and cancer type is likely explained by the fact that Hispanics have a higher incident rate of cancers of the stomach; cervix; and gallbladder, liver, and intrahepatic bile duct..$^{33}$ Those were the three most common types of rare cancers in this study.

\section{Rare cancer patients}

At study entry, patients with rare cancers were as likely as those with common cancers to be treated in a tertiary cancer center, enrolled in a drug trial, or receive palliative care (Table 3 ). Also, at study entry, 350 cancer patients were receiving radiation and/or chemotherapy. Patients with rare cancers were more likely to be receiving both radiation and chemotherapy

Table 1. Types of cancers classified as rare in the study sample $(\mathrm{N}=195)$.

\begin{tabular}{lcc} 
Rare Cancers & N & $\%$ \\
Gastroesophageal & 37 & 19.0 \\
Ovarian and cervical & 30 & 15.4 \\
\hline Hepatocellular, biliary, and gallbladder & 26 & 13.3 \\
Head and neck & 22 & 11.3 \\
\hline Sarcoma and gastrointestinal stromal tumor & 21 & 10.8 \\
Leukemia, multiple myeloma, and Hodgkin lymphoma & 20 & 10.3 \\
\hline Central nervous system & 15 & 7.7 \\
All other sites & 24 & 12.3 \\
\hline
\end{tabular}


than were those with common cancers $(10.3 \%$ vs $3.3 \%$ respectively, $\mathrm{uOR} 3.45, \mathrm{P}=0.01$ ). In a logistic regression analysis, patients with rare cancers remained four times more likely to receive both radiation and chemotherapy at study entry (aOR 4.31, $\mathrm{P}=0.003$ ).

Patients with rare cancers were no more likely than were those with common cancers to acknowledge their terminal illness (33.7\% vs $40.7 \%$, respectively; Table 3 ), participate in advance care planning (57.6\% vs $60.1 \%)$, or have an EOL conversation with their physician (27.7\% vs $34.9 \%)$. Their preferences for care, the EOL care received, or the location of death did not differ significantly (results not shown).

\section{Caregivers of rare cancer patients}

There were no significant differences in the socio-demographic characteristics or baseline health of caregivers of rare cancer patients versus common cancer patients (results not shown). While rare cancer caregivers did not report significantly more emotional distress or mental health diagnoses at study entry, they were more likely than common cancer caregivers to report a decline in health during the final year $(22.1 \%$ vs $15.7 \%$ respectively, $\mathrm{P}=0.05)$ and marginally more likely to report using mental health services to cope with the patient's illness $(22.3 \%$ vs $16.5 \%$ respectively, $\mathrm{P}=0.08)$.

Caregivers of patients with rare vs. common cancers reported no difference in how much information their physicians provided (results not shown). Fewer than 5\% of both rare and common cancer caregivers mentioned wanting more information about the cancer, other forms of treatment or clinical trials, or improved communication with consulting physicians in response to the open-ended question regarding caregiver needs. One caregiver of a patient with a rare cancer reported wishing the patient could help others with the same condition.

Rare cancer caregivers were marginally more likely than common cancer caregivers to prefer that the patient choose treatment focusing on extending life rather than on relieving pain ( $20.8 \%$ vs $14.4 \%$ respectively, $\mathrm{P}=0.08$ ) and significantly more likely to score higher on a scale of optimism [mean 25.5, standard deviation (SD 4.2) vs 24.6 (SD 4.5) respectively, $\mathrm{P}=0.02$ ]

\section{Discussion and Conclusions}

This study investigated differences in the EOL experience of advanced cancer patients dying of rare versus common cancers, and their caregivers. We found that patients with rare cancers were no more likely to acknowledge their illness was terminal, have EOL discussions, or participate in advance care planning than were patients with common cancers. However, patients with rare cancers were four times more likely than were those with common cancers to be receiving both radiation and chemotherapy at study entry. This result deserves further study in light of the entry criteria for the CWC study (i.e. advanced cancer with failure of first line chemotherapy), the frequent lack of proven therapeutic options for rare cancers, and the similarity in rates of patient and caregiver preferences for intensive EOL care by cancer type. Future research should investigate the role of physicians and patients in determining EOL care for those with rare cancers as well as the natural history of their experience (e.g. diagnostic delays, stage at diagnosis, coping, and satisfaction) to improve our understanding of high-quality or optimal care of terminally ill patients with rare cancers.

Also of interest is the finding that caregivers of those with rare cancers did not express greater need for information and may use optimism more often than do caregivers of patients with common cancers as a way to cope. The need for information may be due to the time since diagnosis for this study population. ${ }^{34}$ With respect to coping, the rarity of the cancer itself may lead to less certain predictions of treatment outcomes - and therefore more hope that an unexpected (positive) result may occur. Future research should investigate this possibility and its potential effect on caregiver burden and bereavement adjustment in caregivers of patients with rare versus common cancers, particularly in light of our finding that rare cancer caregivers selfreported worsening health after the patient's diagnosis.

This study has several limitations. First, rare cancers were slightly overrepresented in the sample. This may stem from greater willingness of rare cancer patients to participate in research or more referral of rare cancer cases to the study - possibly due to a higher

Table 2. Participant characteristics by cancer type (total $\mathrm{N}=618$ ).

\begin{tabular}{lccc}
\hline Patient characteristic & $\begin{array}{c}\text { Rare } \\
\text { N=195 }\end{array}$ & $\begin{array}{c}\text { Common } \\
\mathbf{N}=423\end{array}$ & P \\
Age in years, mean (SD) & $57.7(14.3)$ & $60.7(12.3)$ & 0.01 \\
Sex, male, N (\%) & $89(45.6)$ & $220(52.0)$ & 0.14 \\
\hline Race or ethnicity, N (\%) & & \\
$\quad$ White & $126(64.6)$ & $313(74.0)$ & 0.002 \\
Non-white & $32(16.4)$ & $72(17.0)$ & \\
Hispanic & $37(19.0)$ & $38(9.0)$ & \\
Married, N (\%) & $124(64.6)$ & $264(63.2)$ & 0.73 \\
\hline Education in years, mean (SD) & $12.5(4.3)$ & $12.8(3.8)$ & 0.47 \\
Uninsured, N (\%) & $62(32.8)$ & $122(29.3)$ & 0.39 \\
\hline Karnofsky performance score, mean (SD) & $65.1(19.4)$ & $65.7(18.0)$ & 0.69 \\
Charlson comorbidity index, mean (SD) & $5.9(2.4)$ & $6.5(2.3)$ & 0.004 \\
\hline SCID diagnosis, N (\%) & $24(12.6)$ & $51(12.5)$ & 0.97 \\
McGill quality of life, mean (SD) & & & \\
Physical domain & $5.4(1.3)$ & $5.5(1.3)$ & 0.42 \\
Psychological domain & $2.9(2.6)$ & $2.7(2.6)$ & 0.47 \\
Existential domain & $7.6(1.8)$ & $7.7(1.7)$ & 0.41
\end{tabular}

Table 3. Baseline care, terminal illness awareness, and end-of-life planning for patients with rare versus common cancers $(\mathrm{N}=618)$.

\begin{tabular}{lccc}
\hline Baseline care & Rare $(\mathbf{N}=195)$ & Common (N=423) & P \\
Tertiary center & $87(44.6)$ & $159(38.0)$ & 0.12 \\
Drug trial & $22(12.5)$ & $34(8.7)$ & 0.20 \\
\hline Palliative care & $79(42.5)$ & $150(38.0)$ & 0.30 \\
Chemotherapy and radiation* & $11(10.3)$ & $8(3.3)$ & 0.007 \\
\hline Terminal illness acknowledgment & $64(33.7)$ & $169(40.7)$ & 0.10 \\
EOL planning & & & \\
DNR order & $68(36.0)$ & $165(40.3)$ & 0.31 \\
ACP & $110(57.6)$ & $252(60.1)$ & 0.55 \\
EOL discussion & $52(27.7)$ & $145(34.9)$ & 0.08 \\
Preference for intensive EOL care & $51(29.7)$ & $108(28.5)$ & 0.78 \\
\hline
\end{tabular}

Missing data: tertiary center (4), drug trial (48), palliative care (37), terminal illness awareness (13). DNR, Do Not Resuscitate (20); ACP, Advance Care Planning (8); EOL, End of Life discussion (14); preference (67). *Of those patients receiving chemotherapy, radiation or both at study entry; the reference group is chemotherapy or radiation alone $(\mathrm{N}=350)$. 
death rate among this subset of rare cancers, a greater awareness of these cases on the part of referring oncologists or higher referral rate to the predominantly academic institutions represented in the study. We do not know whether differences existed in the type of cancer of participants versus non-participants. Second, the CWC study was not originally designed to investigate differences in the EOL experience or needs of patients with rare cancers compared to those with common cancers. Therefore, it may not be able to address important aspects of the rare cancer experience. For example, CWC cannot answer in sufficient detail whether differences exist in doctorpatient communication (the underpinning of our hypotheses for the study), patient information seeking, or use of alternative treatments. Also, our categorization of tertiary versus nontertiary cancer centers was not based on an empirical comparison of the rate of referred versus primary cancer patients.

Finally, our study also cannot answer whether having a rare cancer is an opportunity for advocacy that serves as a source of hope and meaning in the face of death; or, alternatively, a burden (or both) for patients and families. Anecdotally, rare cancer patients and family members frequently become the organizational core for on- and off-line information, advocacy, and scientific collaboration. . $5,18,21,22^{2}$ Similarly, citizen-created Internet resources, including chat rooms and specialty sites, provide information, support, and empowerment directly to patients with rare conditions who may be geographically dispersed. ${ }^{19,34,35}$ While these opportunities stand separate from physicians and researchers, they also appear to present novel opportunities for recruitment and scientific study. ${ }^{35}$

Despite its limitations, this study is the first investigation we are aware of that explores both the EOL experience of patients with rare cancers and their caregivers as a group and highlights opportunities for further research in the psychosocial and behavioral domains of the rare cancer patient's experience to improve their care and that of their families.

\section{References}

1. Iribarne A. Orphan diseases and adoptive initiatives. JAMA 2003;290:116.

2. Ferrari A, Bisogno G, De Salvo G, et al. The challenge of very rare tumours in childhood: the Italian TREP project. Eur J Cancer 2007;43:654-9.

3. Mano MS, Arai RJ, Hoff PMG. Rare tumors research in emerging countries. Rare Tumors 2010;2:e49.
4. Young JL WK, Ries GLA. Cancers of rare sites. In: National Cancer Institute SP, ed. SEER survival monograph: cancer survival among adults: US SEER Program, 19882001, Patient and Tumor Characteristics. Available from: http://seer.cancer.gov/ archive/publications/survival/

5. Greenlee RR, Goodman MT, Lynch CF, et al. The occurrence of rare cancers in U.S. adults, 1995-2004. Publ Health Rep 2010; 125:28-43.

6. Gatta G, Ciccolallo L, Kunkler I, et al. Survival from rare cancer in adults: a population-based study. Lancet Oncol 2006;7: 132-40.

7. Tan S, Dear K, Bruzzi P, Machin D. Strategy for randomised clinical trials in rare cancers. BMJ 2003;327:47-9.

8. Margo CE. The collaborative ocular melanoma study: an overview. Cancer Control 2004;11:304-9.

9. Seoane-Vazquez E, Rodriguez-Monguio R, Szeinbach S, Visaria J. Incentives for orphan drug research and development in the United States. Orphanet J Rare Dis 2008;3:33.

10. Stakisaitis D, Spokiene I, Juskevicius J, et al. Access to information supporting availability of medicines for patients suffering from rare diseases looking for possible treatments: the EuOrphan Service. Medicina (Kaunas) 2007;43:441-6.

11. Bulusu VR, Fullarton J, Leahy M, et al. Rationale and design of a UK database for a rare cancer type: the GEM Registry for gastrointestinal stromal tumours. Br J Cancer 2013;109:1403-7.

12. Keat N, Law K, Seymour M, et al. International rare cancers initiative. 2013. Available from: http///ecancer.org/journal/ editorial/20-international-rare-cancers-initiative-irci.php.

13. Putkowski S. NORD. A beacon of hope for people with rare disorders. Rare Tumors 2010; 13.

14. Schaefer R. Rare Cancers Europe: joining forces to tackle a common problem. Rare Tumors 2012;4:e24.

15. Anonymous. Very rare cancers-a problem neglected. Lancet Oncol 2001;2:189.

16. Stolk P, Willemen M, Leufkens H. Rare essentials: drugs for rare diseases as essential medicines. Bull World Health Organ 2006;84:745-51.

17. Drummond M, Evans B, LeLorier J, et al. Evidence and values: requirements for public reimbursement of drugs for rare diseases-a case study in oncology. Can J Clin Pharmacol 2009;16:e273-81.

18. Reynolds T. Celebrity's death spurs interest in rare cancers. J Natl Cancer Inst 1999;91:2070-1.

19. Patsos M. MSJAMA: the Internet and medicine: building a community for patients with rare diseases. JAMA 2001;285:805.

20. Fernandez CV. Our moral obligations in caring for patients with orphan cancers. CMAJ 2007;176:297.

21. Foster J. Insider Research with family members who have a member living with rare cancer. Int J Qual Methods 2009;8:16-26.

22. Marcus $\mathrm{AD}$. The loneliness of fighting a rare cancer. Health Aff 2010;29:203-6.

23. Politi M, Han P, Col N. Communicating the uncertainty of harms and benefits of medical interventions. Med Decis Making 2007;27:681-95.

24. Griffiths J, Willard C, Burgess A, et al. Meeting the ongoing needs of survivors of rarer cancer. Eur J Oncol Nurs 2007;11: 434-41.

25. Wright AA ZB, Ray A, et al. Associations between end-of-life discussions, patient mental health, medical care near death, and caregiver bereavement adjustment. JAMA 2008;300:1665-73.

26. National Cancer Institute. Common cancer types. 2013. Available from: http:/www.cancer.gov/cancertopics/types/commoncancers.

27. Williams J, Gibbon M, First M, et al. The structured clinical interview for DSM-III-R (SCID). II. Multisite test-retest reliability. Arch Gen Psychiatry 1992;49:630-6.

28. First M, Spitzer R, Gibbon M, Williams J. Structured clinical interview for the DSMIV axis I disorders - Patient Edition (SCIDI/P). Version 2.0 ed. New York: Biometrics Research Department, New York State Psychiatric Institute; 1995.

29. Charlson M, Pompei P, Ales K, MacKenzie C. A new method of classifying prognostic comorbidity in longitudinal studies: development and validation. J Chronic Dis 1987;40:373-83.

30. Schag C, Heinrich R, Ganz P. Karnofsky performance status revisited: reliability, validity, and guidelines. J Clin Oncol 1984; 2:187-93.

31. Yates J, Chalmer B, McKegney F. Evaluation of patients with advanced cancer using the Karnofsky performance status. Cancer 1980;45:2220-4.

32. Ware JE SC. The MOS 36-item short-form health survey (SF-36). I. Conceptual framework and item selection. Medical Care 1992;30:473-83.

33 . American Cancer Society. Cancer Facts \& Figures for Hispancis/Latinos 2012-2014. Atlanta: American Cancer Society, 2012.

34. Lasker J, Sogolow E, Sharim R. The role of an online community for people with a rare disease: content analysis of messages posted on a primary biliary cirrhosis mailinglist. J Med Internet Res 2005;7:e10.

35. Bedgood R, Sadurski R, Schade R. The use of the internet in data assimilation in rare diseases. Dig Dis Sci 2007;52:307-12. 